T. AMATOV, N. TSUJI, R. MAJI, L. SCHREYER, H. ZHOU, M. LEUTZSCH, B. LIST* (MAXPLANCK-INSTITUT FÜR KOHLENFORSCHUNG, MÜLHEIM AN DER RUHR, GERMANY AND HOKKAIDO UNIVERSITY, SAPPORO, JAPAN)

Confinement-Controlled, either syn- or anti-Selective Catalytic Asymmetric Mukaiyama Aldolizations of Propionaldehyde Enolsilanes

J. Am. Chem. Soc. 2021, 143, 14475-14481, DOI: 10.1021/jacs.1c07447.

\title{
Catalytic Asymmetric Aldolization of Propanal Enol Silanes
}

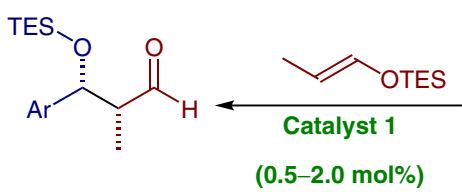

12 examples

$88-97 \%$ yield

dr from $82: 18$ to $99: 1$

er from $93: 7$ to $99: 1$

ected examples:<smiles>C[C@H](C=O)[C@H](OC(F)(F)F)c1ccccc1</smiles>

$97 \%, d r=99: 1$

er $=98: 2$<smiles>Cc1ccccc1[C@@H](OC(F)F)[C@H](C)C=O</smiles>

$97 \%, \mathrm{dr}=81: 19$

er $=97: 3$<smiles>O=CBr</smiles>

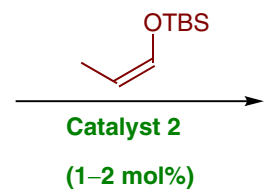

(1-2 mol\%)
TBS<smiles>COC(Br)[C@H](C)C=O</smiles>

12 examples

$37-98 \%$ yield

dr from 89:11 to $99: 1$

er from $87: 13$ to $98.5: 1.5$

Mukaiyama aldol syn-selective anti-selective

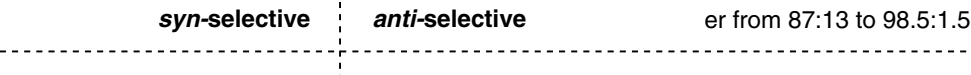<smiles>C[C@@H](C=O)[C@H](OC(F)(F)F)c1cccs1</smiles>

$99 \%, d r=99: 1$

er $=96: 4$<smiles>C[C@H](C=O)[C@H](OC(F)F)c1ccc(Br)cc1</smiles>

$96 \%, d r=99: 1$ er $=98: 2$<smiles>C[C@@H](C=O)C(O[AsH3])c1ccccc1</smiles>

$97 \%, d r=99: 1$

er $=98: 2$<smiles>Cc1ccccc1C(O[AsH3])[C@@H](C)C=O</smiles>

$97 \%, d r=99: 1$ er $=98: 2$<smiles>CC(C=O)C(O[13CH3])c1cccs1</smiles>

$97 \%, d r=99: 1$

er $=93: 7$<smiles>C[C@@H](C=O)C(O[AsH3])c1ccc(Br)cc1</smiles>

$97 \%, d r=99: 1$

er $=98: 2$

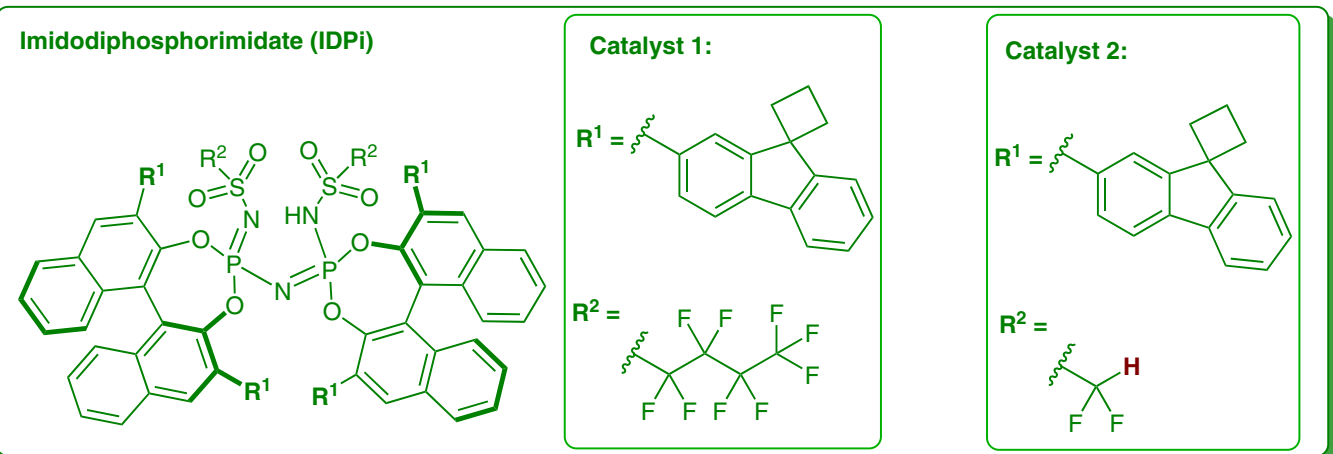

Significance: List and co-workers report an asymmetric Mukaiyama aldolization controlled by a confined chiral imidodiphosphorimidate catalyst. By using catalysts 1 and 2, syn- or anti-aldol products can be obtained from ( $E$ )- or (Z)-enolsilanes, respectively, in high yields and with high diastereo- and enantioselectivities.
Comment: Compared with the reported pioneering work (S. E. Denmark, S. K. Ghosh Angew. Chem. Int. Ed. 2001, 40, 4759), this method presents an improved, fully atom- and step-economic strategy. Ultimately, it could streamline the syntheses of complex oligopropionates.
Key words

confinement

asymmetric catalysis

stereoselectivity

Mukaiyama reaction 